\title{
Photonic nanowires and waveguides for spectral shaping with Sagnac loop reflectors
}

This paper was downloaded from TechRxiv (https://www.techrxiv.org).

\section{LICENSE}

CC BY 4.0

SUBMISSION DATE / POSTED DATE

29-01-2022 / 01-02-2022

\section{CITATION}

Moss, David (2022): Photonic nanowires and waveguides for spectral shaping with Sagnac loop reflectors. TechRxiv. Preprint. https://doi.org/10.36227/techrxiv.19091273.v1

$\mathrm{DOI}$

10.36227/techrxiv.19091273.v1 


\title{
Photonic nanowires and waveguides for spectral shaping with Sagnac loop reflectors
}

\author{
David J. Moss \\ Optical Sciences Centre, Swinburne University of Technology, Hawthorn, VIC 3122, Australia.
}

\begin{abstract}
We propose and theoretically investigate integrated photonic filters based on two coupled Sagnac loop reflectors (SLRs) formed by a self-coupled optical waveguide. Recently we investigated integrated photonic filters based on cascaded SLRs and coupled SLRs. Here, we advance this field by presenting a unique approach of using coupled SLRs formed by a self-coupled optical waveguide. This enables us to achieve high performance filter functions including Fano-like resonances and wavelength interleaving with a simpler design and a higher fabrication tolerance by tailoring coherent mode interference in the device. Our design takes into account the device fabrication issues as well as the requirements for practical applications. As a guide for practical device fabrication, an analysis of the impact of the structural parameters and fabrication tolerance on each filter function is also provided. The Fano-like resonances show a low insertion loss (IL) of $1.1 \mathrm{~dB}$, a high extinction ratio of $30.2 \mathrm{~dB}$, and a high slope rate (SR) of $747.64 \mathrm{~dB} / \mathrm{nm}$. The combination of low IL and high SR promises this device for Fano resonance applications. Our device also can achieve wavelength de-interleaving function with high fabrication tolerance which is attractive for optical interleavers that need a flat-top symmetric filter shape. Optical interleavers and de-interleavers are core elements for signal multiplexing and demultiplexing in wavelength division multiplexing optical communication systems. Versatile spectral responses with a simple design, compact device footprint, and high fabrication tolerance make this approach highly promising for flexible response shaping in a wide variety of applications.
\end{abstract}

Keywords: Integrated photonic resonators, Sagnac loop reflectors, Fano resonance, interleavers.

\section{INTRODUCTION}

Integrated photonic resonators (IPRs) have allowed a variety of functional optical devices, including filters, modulators, sensors, switches, and logic gates, thanks to their compact footprint, flexible topology, and great scalability [1-4]. Compared to the IPRS formed by photonic crystal structures [5] and gratings [6] that have sub-wavelength cavity lengths, the IPRs formed by directional-coupled wire waveguides with longer cavity lengths have smaller free spectral ranges (FSRs) that match the spectral grids of state-of-the-art wavelength division multiplexing (WDM) optical communication systems, thus making them more widely applicable to these systems. In addition, sub-wavelength dimensions of photonic crystal cavity and Bragg grating structures are more prone to fabrication tolerances as compared with directional-coupled wire waveguides. Ring resonators (RRs), and Sagnac loop reflectors (SLRs), which are essential building blocks for IPRs, are made up of directional couplers. Unlike RRs, which only allow for unidirectional light propagation, SLRs allow for bidirectional light propagation as well as mutual coupling between light travelling in opposing directions, resulting in a more versatile coherent mode interference and spectral response. Furthermore, a standing-wave (SW) resonator made up of cascaded SLRs has a cavity length about half that of a traveling wave (TW) resonator made up of a ring resonator with the same FSR, allowing for a more compact device footprint.

We investigated integrated photonic filters based on cascaded SLRs $[7,8]$ and coupled SLRs $[9,10]$ in our previous work. Here, we advance this field by presenting the novel approach of using two coupled SLRs with a feedback loop formed by a self-coupled wire waveguide that yield different response shapes including Fano-like resonances and wavelength de-interleaving [11].

In our design, we take into account the device fabrication issues experienced in Refs. [7, 8] as well as the needs for practical applications. As a guide for practical device fabrication, an analysis of the influence of structural parameters and fabrication tolerance is also provided. 


\section{DEVICE CONFIGURATION}

The proposed structure is illustrated schematically in Fig. 1, which consists of two inverse-coupled SLRs with a feedback loop formed by a single self-coupled wire waveguide. Table 1 details the device's structural parameters. To simplify the discussion, we assume that $L_{\mathrm{SLR} 1}=L_{\mathrm{SLR} 2}=L_{\mathrm{SLR}}$. The spectral response of the device is calculated using the scattering matrix method [7,9]. In the device model, we use waveguide group index of $n_{g}=4.3350$ (transverse electric (TE) mode) and propagation loss of $\alpha=55 \mathrm{~m}^{-1}$ (i.e., $2.4 \mathrm{~dB} / \mathrm{cm}$ ), which are in line with our previously fabricated siliconon-insulator (SOI) devices $[7,8,12]$. The device is designed based on, but not limited to, the SOI platform.

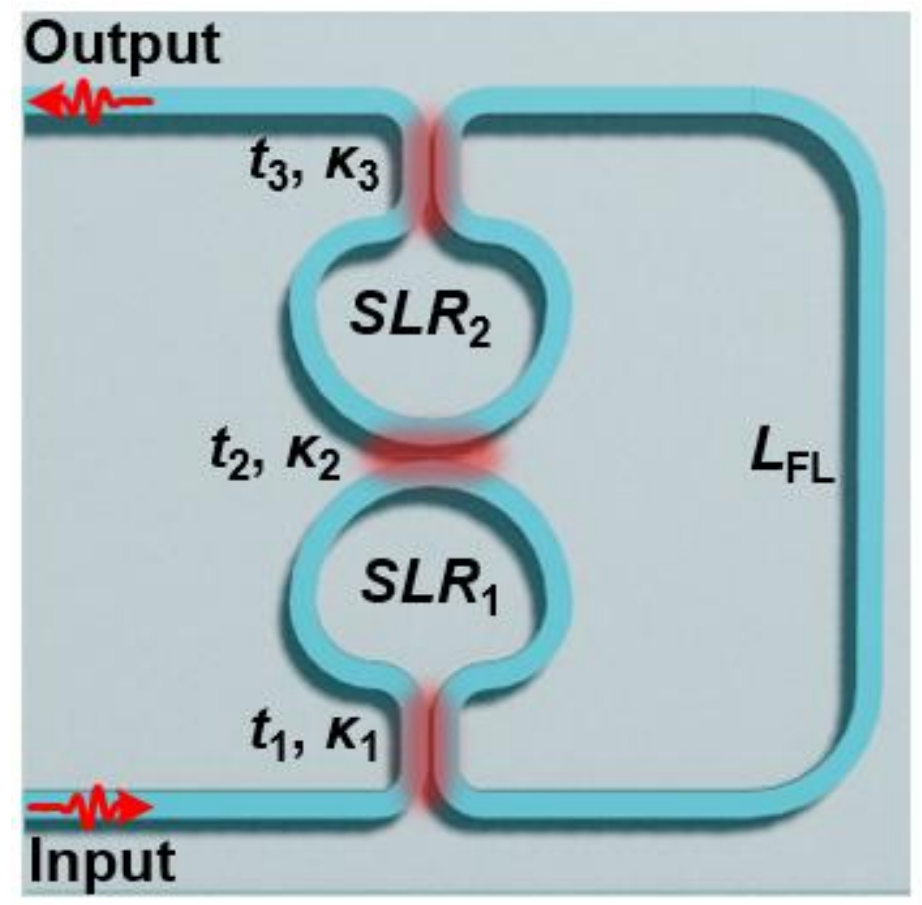

Figure 1. Schematic configuration of device. The definitions of $t_{i}(i=1,2,3), L_{\mathrm{SLR} i}(i=1,2)$, and $L_{\mathrm{FL}}$ are given in Table 1.

Table 1. Definitions of device structural parameters

\begin{tabular}{|c|c|c|c|}
\hline Waveguides & Length & Transmission factor & Phase shift ${ }^{\text {b }}$ \\
\hline Feedback loop between SLRs $(i=1,2)$ & $L_{\mathrm{FL}}$ & $a_{\mathrm{f}}$ & $\varphi_{\mathrm{f}}$ \\
\hline Sagnac loop in $S L R_{i}(i=1,2)$ & LSLRi & $a_{\mathrm{si}}$ & $\varphi_{\mathrm{si}}$ \\
\hline Directional couplers & \multicolumn{2}{|c|}{ Field transmission coefficient ${ }^{\mathrm{c}}$} & Field cross-coupling coefficient $^{\mathrm{c}}$ \\
\hline Coupler in SLRs $(i=1,2)$ & \multicolumn{2}{|r|}{$t_{i}$} & $\kappa_{i}$ \\
\hline Coupler between $S L R_{\mathrm{S}}$ & \multicolumn{2}{|r|}{$t_{2}$} & $\kappa_{2}$ \\
\hline
\end{tabular}

${ }^{\mathrm{a}} a_{\mathrm{f}}=\exp \left(-\alpha L_{\mathrm{FL}} / 2\right), a_{\mathrm{si}}=\exp \left(-\alpha L_{\mathrm{SLR} i} / 2\right), \alpha$ is the power propagation loss factor.

${ }^{\mathrm{b}} \varphi_{\mathrm{f}}=2 \pi n_{\mathrm{g}} L_{\mathrm{FL}} / \lambda, \varphi_{\mathrm{si}}=2 \pi n_{\mathrm{g}} L_{\mathrm{SLR} i} / \lambda, n_{\mathrm{g}}$ is the group index and $\lambda$ is the wavelength.

${ }^{\mathrm{c}} t_{i}^{2}+\kappa_{i}^{2}=1$ for lossless coupling are assumed for all the directional couplers

In the following sections, mode interference in the device is tailored to achieve high-performance filtering functions, including Fano-like resonances and wavelength de-interleaving.

\section{FANO-LIKE RESONANCES}

Fano resonances are a fundamental physical phenomenon demonstrating an asymmetric spectral lineshape arising from quantum interference between discrete and continuum states $[13,14]$. They have underpinned many applications such as 
optical switching, data storage, sensing, and topological optics, due to their unique physics and capability of providing ultra-narrow spectral linewidths [13-15]. In this section, the spectral response of the device in Fig. 1 is tailored to realize Fano-like resonances with high slope rates (SRs) and low insertion loss (IL). The power transmission and reflection spectra is depicted in Fig. 2(a). The device structural parameters are $L_{\mathrm{SLR}}=100 \mu \mathrm{m}, L_{\mathrm{FL}}=300 \mu \mathrm{m}, t_{1}=t_{3}=0.82$, and $t_{2}$ $=0.92$. Clearly, there are periodical Fano resonances with identical asymmetric resonant lineshape in each period at output port. The high uniformity of the response shape of the resonator could be suitable for applications in WDM systems. A zoom-in view of Fig. 2(a) is shown in Fig. 2(b), together with another curve showing the corresponding result for another device with the same structural parameters except for a different $t_{2}=1$. As can be seen, when $t_{2}=1$, there is no Fano resonance, distinguishing between the device in Fig. 1 and the two cascaded SLRs in Ref. [16]. The Fano resonances in Fig. 2(a) show a high extinction ratio (ER) of $30.2 \mathrm{~dB}$ and a high SR (defined as the ratio of the ER to the wavelength difference between the resonance peak and notch) of $747.64 \mathrm{~dB} / \mathrm{nm}$.
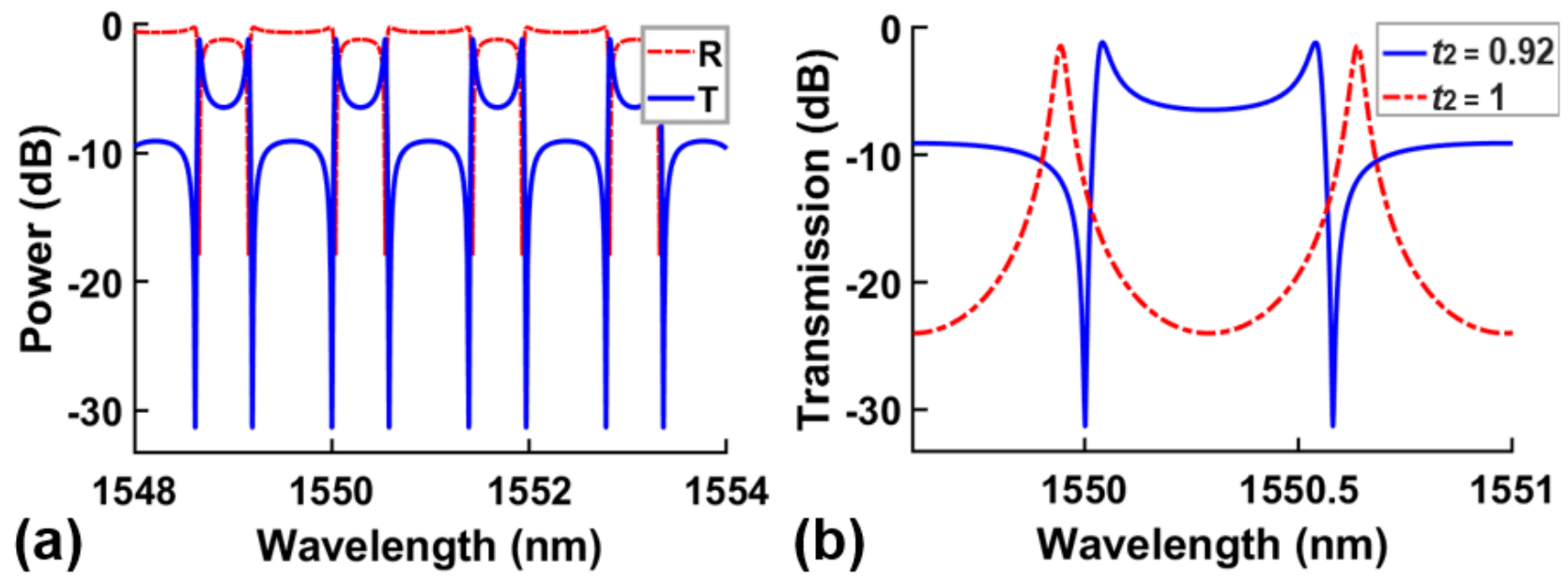

Figure 2. (a) Power transmission and reflection spectra when $L_{\mathrm{SLR}}=100 \mu \mathrm{m}, L_{\mathrm{FL}}=300 \mu \mathrm{m}, t_{1}=t_{3}=0.82$, and $t_{2}=0.92$. $\mathrm{T}$ : Transmission spectrum at output port. R: reflection spectrum at input port. (b) Power transmission spectra at output port for $t_{2}=0.92$ and $t_{2}=1$. In (b), the structural parameters are kept the same as those in (a) except for $t_{2}$.

The performance of the Fano-like resonances generated by the coupled SLRs in our prior work [9, 10] and the device in Fig. 1 are compared in Table 2. For comparison, the device structural parameters $\left(L_{\mathrm{SLR} i}, n_{\mathrm{g}}\right.$, and $\left.\alpha\right)$ of all the three structures were kept the same except for the transmission coefficients $\left(t_{i}\right)$ that were tuned to obtain the highest SR for each structure. As compared with previous devices, the device presented here has a much lower IL of $1.1 \mathrm{~dB}$, along with a slightly improved SR. The combination of high SR and low IL promises this device for Fano resonance applications. We note that a low IL of $1.1 \mathrm{~dB}$ is outstanding among the reported Fano-resonance devices on the SOI platform [17, 18], which makes the device here more attractive for practical applications.

Table 2. Performance comparison of Fano-like resonances generated by different SLR-based devices.

\begin{tabular}{c|c|c|c|c|c}
\hline \hline Device structure & IL $(\mathbf{d B})$ & ER (dB) & SR (dB/nm) & FSR (GHz) & Ref. \\
\hline Two parallel WC-SLRs $^{\text {a }}$ & 6.3 & 13.9 & 389 & 692.02 & {$[9]$} \\
\hline Three zig-zag WC-SLRs $^{\mathbf{b}}$ & 3.7 & 63.4 & 721.28 & 230.68 & {$[10]$} \\
\hline Device in Fig. 1 & 1.1 & 30.2 & 747.64 & 173 & This work \\
\hline \hline
\end{tabular}

aC-SLRs: waveguide coupled SLRs.

${ }^{\mathrm{b}}$ For comparison, the length of the SLRs $\left(L_{\mathrm{SLR} i}, i=1-3\right)$ and the connecting waveguide $\left(L_{i}, i=1-4\right)$ is slightly changed from $115 \mu \mathrm{m}$ in [10] to $100 \mu \mathrm{m}$.

In Figs. 3(a)-(c), we further investigate the impact of the device structural parameters including $t_{i}(i=1-3)$ and length variations of feedback loop $\left(\Delta L_{\mathrm{FL}}\right)$ on the performance of the Fano resonance. In each figure, we changed only one structural parameter, keeping the others the same as those in Fig. 2 (a). In Figs. 3(a)-(c), (i) shows power transmission spectra and (ii) shows the corresponding IL and SR for different $t_{i}(i=1-3)$, and $\Delta L_{\mathrm{FL}}$, respectively. The SR decreases with $t_{i}(i=1,3)$, while the IL first decreases with $t_{i}(i=1,3)$ and then remains almost unchanged. The SR decreases with $t_{2}$, while the IL shows an opposite trend, reflecting that both of the two parameters can be improved by enhancing the 
coupling strength between SLR 1 and $\mathrm{SLR}_{2}$. As shown in Fig. 3(c), the filter shape remains unchanged while the Fanolike resonance peak redshifts as $\Delta L_{\mathrm{FL}}$ increases. This indicates that the resonance wavelengths can be tuned by introducing thermo-optic micro-heaters [18] or carrier-injection electrodes [19] along feedback loop to tune the phase shift.
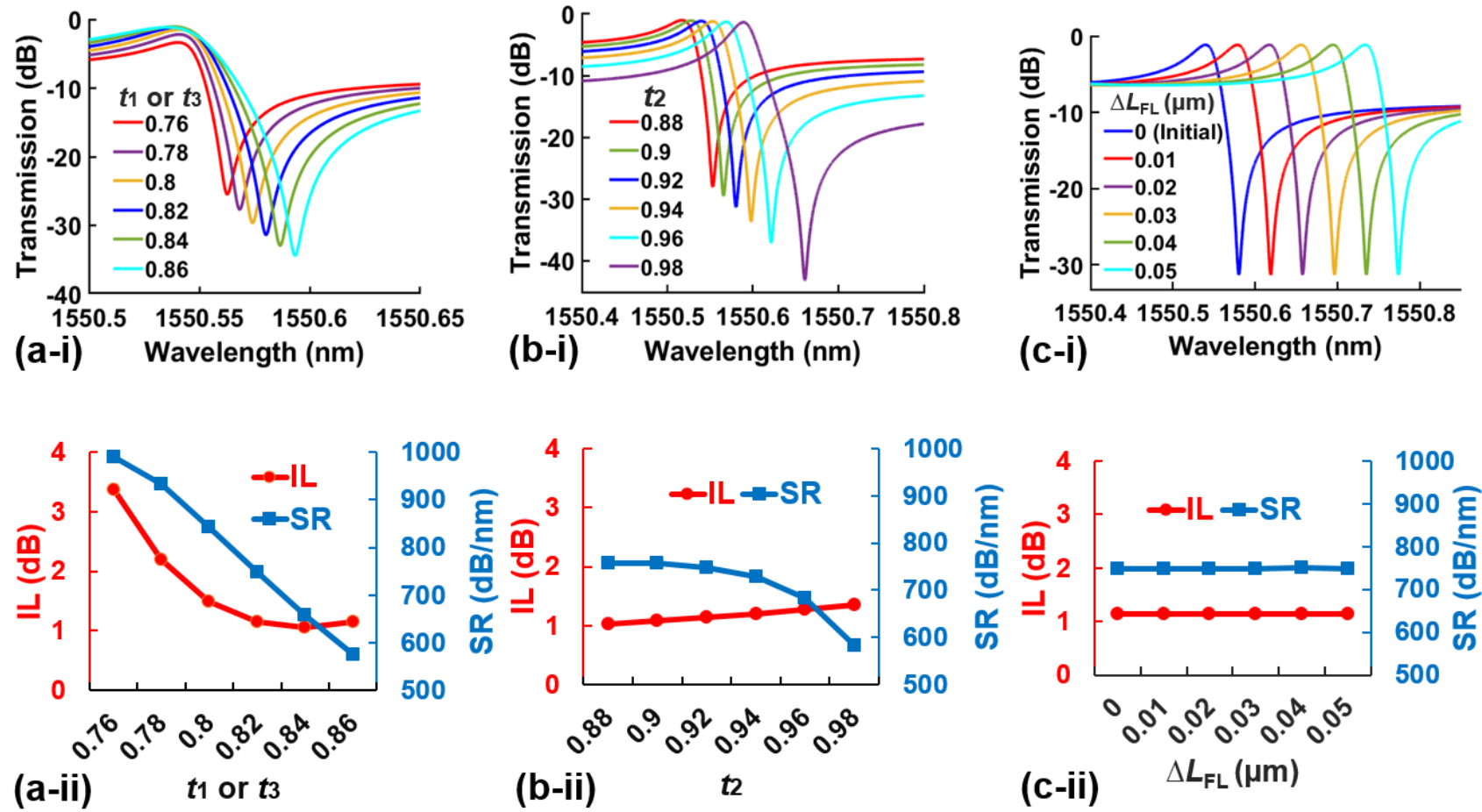

Figure 3. (a)-(c) Power transmission spectra (i) and the corresponding IL and SR (ii) for different $t_{i}(i=1-3)$ and $\Delta L$ FL respectively. In (a)-(c), the structural parameters are kept the same as those in Fig. 2(a) except for the varied parameters.

\section{WAVELENGTH DE-INTERLEAVING FUNCTION}

Optical interleavers and de-interleavers are core elements for signal multiplexing/demultiplexing in wavelength division multiplexing (WDM) optical communication systems [20, 21]. In this section, we engineer the spectral response of the device in Fig. 1 to achieve wavelength de-interleaving function. Flat-top spectral response of de-interleavers minimize the filtering distortions and group delay variation and high ER minimize signal crosstalk between adjacent channels [22]. Fig. 4(a) shows the power transmission and reflection spectra when the device structural parameters are $L_{\mathrm{SLR}}=100 \mu \mathrm{m}$, $L_{\mathrm{FL}}=300 \mu \mathrm{m}, t_{1}=0.992$, and $t_{2}=t_{3}=0.95$. The IL, ER, and 3-dB bandwidth for the passband at output port are $0.36 \mathrm{~dB}$, $12.7 \mathrm{~dB}$, and $83.65 \mathrm{GHz}$, respectively. The IL, ER, and 3-dB bandwidth for the reflection spectrum at input port are 0.33 $\mathrm{dB}, 12 \mathrm{~dB}$, and $91.9 \mathrm{GHz}$, respectively. As compared with flat-top filters based on cascaded ring resonators [23], ringassisted Mach-Zehnder interferometers [24], and cascaded SLRs [7], our device can achieve the same level of filtering flatness with fewer subunits.

We further investigate the impact of varied $t_{i}(i=1-3)$ in Figs. 4(b)-(d), respectively. For simplification, we only show the spectral response at output port. In Fig. 4(b), as $t_{1}$ increases, the ER of the passband decreases while the top flatness improves, reflecting the trade-off between them. In Figs. 4(c)-(d), the bandwidth of the passband increases with $t_{2}$, $t_{3}$, respectively, while the ER shows an opposite trend. 

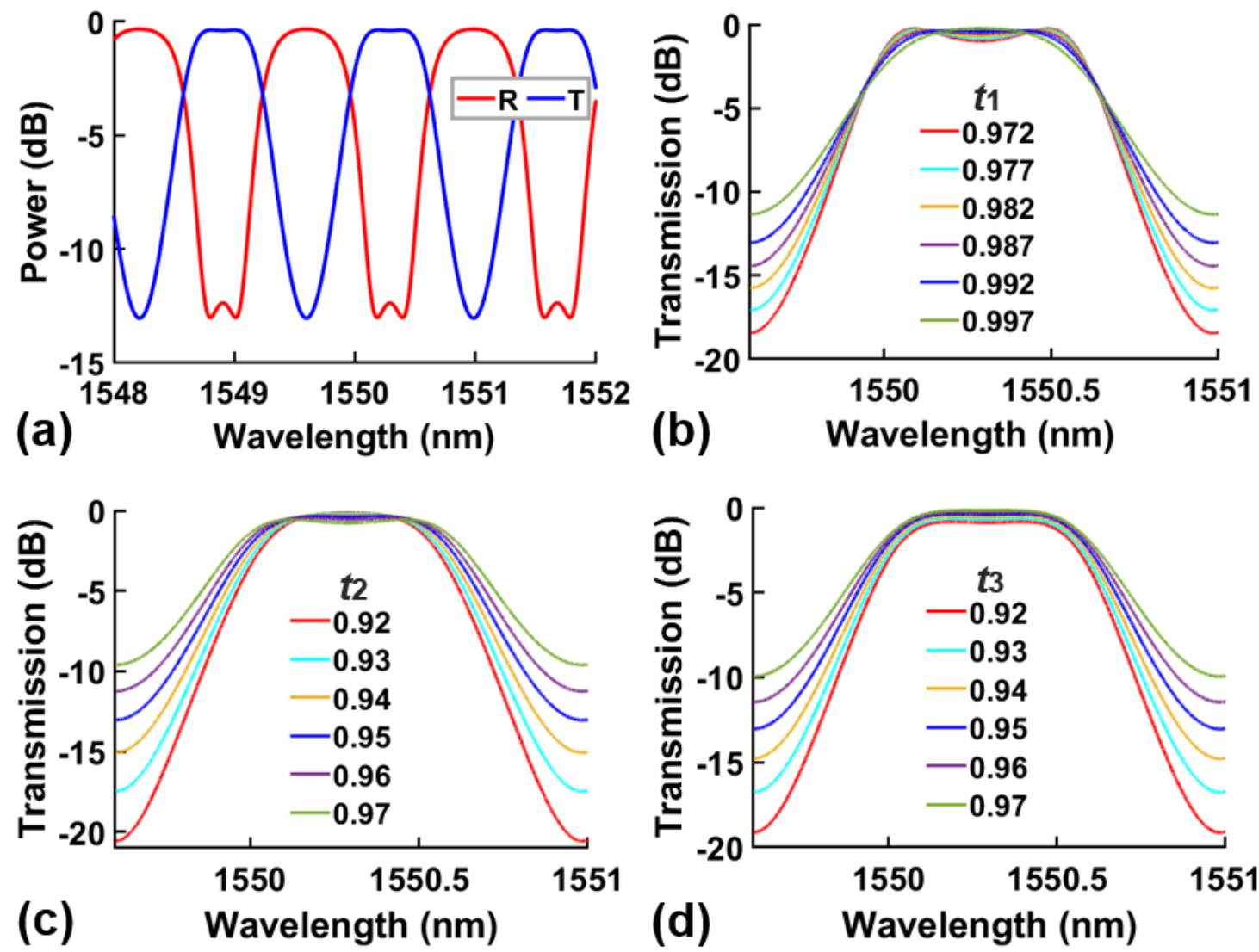

Figure 4. (a) Power transmission and reflection spectra of the device when $L \mathrm{SLR}=100 \mu \mathrm{m}, L_{\mathrm{FL}}=300 \mu \mathrm{m}, t_{1}=0.992$, and $t_{2}=t_{3}=0.95$. T: Transmission spectrum at output port. R: reflection spectrum at input port. (b)-(d) Power transmission spectra for different $t_{i}(i=1-$ 3), respectively. In (b)-(d), the structural parameters are kept the same as those in (a) except for the varied parameters.

We also investigate the impact of varied $\Delta L_{\mathrm{SLR} i}(i=1,2)$ and $\Delta L_{\mathrm{FL}}$ in Figs. 5(a)-(c), respectively. In Figs. 5(a)-(c), as $\Delta L_{\mathrm{SLR} i}(i=1,2)$ or $\Delta L_{\mathrm{FL}}$ increases, the filter shape remains unchanged while the resonance redshifts. Since the resonant cavity of the device is formed by a single self-coupled wire waveguide, random length fabrication errors in different parts (i.e., SLR 1 in Fig. 5(a), SLR 2 in Fig. 5(b), and feedback loop in Fig. 5(c),) will not induce any asymmetry in the filter shape. This yields a higher fabrication tolerance as compared with the coupled SLRs in Refs. [9, 10], which is particularly attractive for optical interleavers that require a flat-top symmetric filter shape.From Figs. 4(b)-(c) and Fig. 5, it can be seen that the slight changes in the structural parameters induced by fabrication disorders have no major impact on device performance.
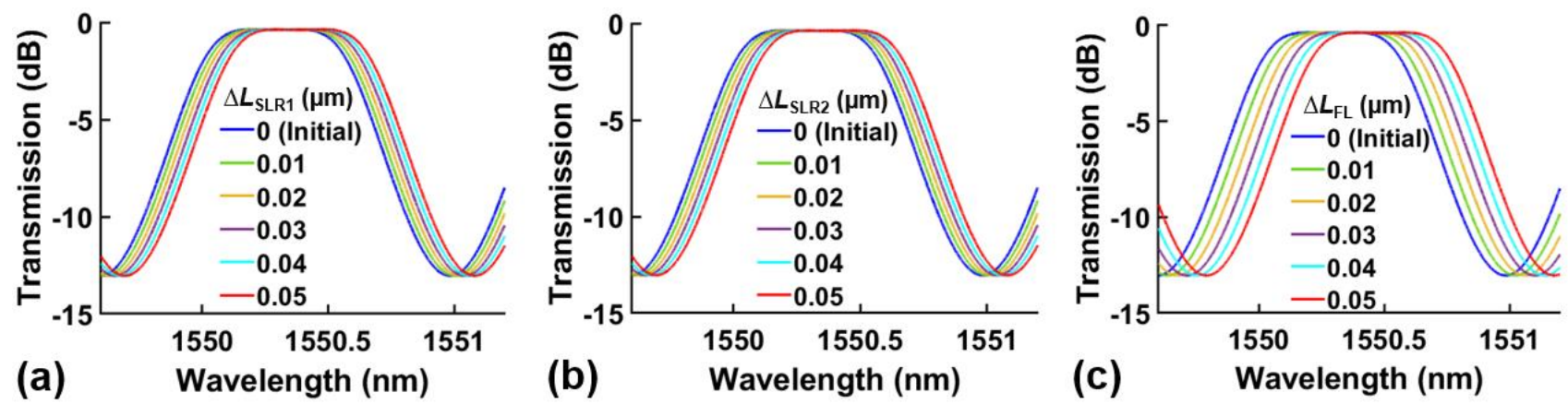

Figure 5. (a)-(c) Power transmission spectra for different $\Delta L_{\mathrm{SLR} i}(i=1,2)$ and $\Delta L_{\mathrm{FL}}$, respectively. In (a)-(c), the structural parameters are kept the same as those in Fig. 4(a) except for the varied parameters. 


\section{CONCLUSIONS}

We have theoretically investigated integrated photonic filters based on two coupled SLRs with a feedback loop formed by a self-coupled optical waveguide. High performance filter functions including Fano-like resonances and wavelength de-interleaving are achieved by tailoring coherent mode interference in the device. Our design takes into account the device fabrication experience as well as the requirements for practical applications. The impact of device structural parameters on each filter function is analyzed to facilitate optimized performance. Versatile spectral responses, compact device footprint, and high fabrication tolerance make this approach highly promising for flexible response shaping in a wide variety of applications including potentially optical microcombs for advanced dispersion design for many applications. [25-145]

\section{REFRENCES}

[1] W. Bogaerts, P. De Heyn, T. Van Vaerenbergh et al., "Silicon microring resonators,” Laser \& Photonics Reviews, 6(1), 47-73 (2012).

[2] Z. Yao, K. Wu, B. X. Tan et al., "Integrated Silicon Photonic Microresonators: Emerging Technologies," IEEE Journal of Selected Topics in Quantum Electronics, 24(6), 1-24 (2018).

[3] S. Feng, T. Lei, H. Chen et al., "Silicon photonics: from a microresonator perspective," Laser \& Photonics Reviews, 6(2), 145-177 (2012).

[4] H. Arianfard, B. Khajeheian, and R. Ghayour, "Tunable band (pass and stop) filters based on plasmonic structures using Kerr-type nonlinear rectangular nanocavity," Optical Engineering, 56(12), 121902 (2016).

[5] T. Gu, N. Petrone, J. F. McMillan et al., "Regenerative oscillation and four-wave mixing in graphene optoelectronics," Nature Photonics, 6(8), 554-559 (2012).

[6] P. Cheben, R. Halir, J. H. Schmid et al., "Subwavelength integrated photonics," Nature, 560(7720), 565-572 (2018).

[7] J. Wu, T. Moein, X. Xu et al., "Advanced photonic filters based on cascaded Sagnac loop reflector resonators in silicon-on-insulator nanowires," APL Photonics, 3(4), 046102 (2018).

[8] J. Wu, T. Moein, X. Xu et al., "Micro-ring resonator quality factor enhancement via an integrated Fabry-Perot cavity," APL Photonics, 2(5), 056103 (2017).

[9] H. Arianfard, J. Wu, S. Juodkazis et al., “Advanced Multi-Functional Integrated Photonic Filters Based on Coupled Sagnac Loop Reflectors,” Journal of Lightwave Technology, 39(5), 1400-1408 (2021).

[10] H. Arianfard, J. Wu, S. Juodkazis et al., "Three Waveguide Coupled Sagnac Loop Reflectors for Advanced Spectral Engineering," Journal of Lightwave Technology, 39(11), 3478-3487 (2021).

[11] H. Arianfard, J. Wu, S. Juodkazis et al., "Spectral Shaping Based on Coupled Sagnac Loop Reflectors Formed by a Self-Coupled Wire Waveguide," IEEE Photonics Technology Letters, 33(13), 680-683 (2021).

[12] R. Petruškevičius, A. Balčytis, D. Urbonas et al., "Microring resonators with circular element inner-wall gratings for enhanced sensing," Japanese Journal of Applied Physics, 59(SO), SOOD02 (2020).

[13] A. E. Miroshnichenko, S. Flach, and Y. S. Kivshar, "Fano resonances in nanoscale structures," Reviews of Modern Physics, 82(3), 2257-2298 (2010).

[14] M. F. Limonov, M. V. Rybin, A. N. Poddubny et al., "Fano resonances in photonics," Nature Photonics, 11, 543 (2017).

[15]E. Kamenetskii, A. Sadreev, and A. Miroshnichenko, [Fano Resonances in Optics and Microwaves: Physics and Applications] Springer International Publishing, (2018).

[16] X. Sun, L. Zhou, J. Xie et al., "Tunable silicon Fabry-Perot comb filters formed by Sagnac loop mirrors," Optics Letters, 38(4), 567-569 (2013).

[17] A. Li, and W. Bogaerts, "An actively controlled silicon ring resonator with a fully tunable Fano resonance," APL Photonics, 2(9), 096101 (2017).

[18] T. Zhao, H. Xiao, Y. Li et al., "Independently tunable double Fano resonances based on waveguide-coupled cavities," Optics Letters, 44(12), 3154-3157 (2019).

[19] M. C. M. M. Souza, G. F. M. Rezende, L. A. M. Barea et al., "Spectral engineering with coupled microcavities: active control of resonant mode-splitting," Optics Letters, 40(14), 3332-3335 (2015).

[20] S. Cao, J. Chen, J. N. Damask et al., "Interleaver technology: comparisons and applications requirements,' Journal of Lightwave Technology, 22(1), 281-289 (2004). 
[21] S. Lai, Z. Xu, B. Liu et al., "Compact silicon photonic interleaver based on a self-coupled optical waveguide," Appl Opt, 55(27), 7550-5 (2016).

[22] P. Dong, N.-N. Feng, D. Feng et al., "GHz-bandwidth optical filters based on high-order silicon ring resonators," Optics Express, 18(23), 23784-23789 (2010).

[23] B. E. Little, S. T. Chu, P. P. Absil et al., "Very high-order microring resonator filters for WDM applications," IEEE Photonics Technology Letters, 16(10), 2263-2265 (2004).

[24] L.-W. Luo, S. Ibrahim, A. Nitkowski et al., "High bandwidth on-chip silicon photonic interleaver," Optics Express, 18(22), 23079-23087 (2010).

[25] X. Xu et al, "Photonic perceptron based on a Kerr microcomb for scalable high speed optical neural networks", Laser and Photonics Reviews, vol. 14, no. 8, 2000070 (2020). DOI: 10.1002/lpor.202000070.

[26] X. Xu, et al., "11 TOPs photonic convolutional accelerator for optical neural networks”, Nature 589, 44-51 (2021).

[27] Feldmann, J. et al., "Parallel convolutional processing using an integrated photonic tensor core", Nature 589, 52-58 (2021).

[28] T. Zhou et al, "Large-scale neuromorphic optoelectronic computing with a reconfigurable diffractive processing unit", Nature Photonics Vol 15, (5) 367 (2021).

[29] B. J. Shastri et al., "Photonics for artificial intelligence and neuromorphic computing", Nature Photonics 15, (2) 102-114 (2021).

[30] Tait, A. N. et al., "Demonstration of WDM weighted addition for principal component analysis", Optics Express 23, 12758-12765 (2015).

[31] Pasquazi, A. et al. Micro-combs: a novel generation of optical sources. Physics Reports 729, 1-81 (2018).

[32] Moss, D. J. et al., "New CMOS-compatible platforms based on silicon nitride and Hydex for nonlinear optics", Nature photonics 7, 597 (2013).

[33] Kippenberg, T. J., Gaeta, A. L., Lipson, M. \& Gorodetsky, M. L. Dissipative Kerr solitons in optical microresonators. Science 361, 567 (2018).

[34] Savchenkov, A. A. et al. Tunable optical frequency comb with a crystalline whispering gallery mode resonator. Physics Review Letters 101, 093902 (2008).

[35] Spencer, D. T. et al. An optical-frequency synthesizer using integrated photonics. Nature 557, 81-85 (2018).

[36] Marin-Palomo, P. et al. Microresonator-based solitons for massively parallel coherent optical communications. Nature 546, 274 (2017).

[37] B. Corcoran, et al., "Ultra-dense optical data transmission over standard fiber with a single chip source", Nature Communications, vol. 11, Article:2568, 2020.

[38] Kues, M. et al. Quantum optical microcombs. Nature Photonics 13, (3) 170-179 (2019). doi:10.1038/s41566019-0363-0

[39] C. Reimer, L. Caspani, M. Clerici, et al., "Integrated frequency comb source of heralded single photons," Optics Express, vol. 22, no. 6, pp. 6535-6546, 2014.

[40] C. Reimer, et al., "Cross-polarized photon-pair generation and bi-chromatically pumped optical parametric oscillation on a chip", Nature Communications, vol. 6, Article 8236, 2015. DOI: 10.1038/ncomms9236.

[41] L. Caspani, C. Reimer, M. Kues, et al., "Multifrequency sources of quantum correlated photon pairs on-chip: a path toward integrated Quantum Frequency Combs," Nanophotonics, vol. 5, no. 2, pp. 351-362, 2016.

[42] C. Reimer et al., "Generation of multiphoton entangled quantum states by means of integrated frequency combs," Science, vol. 351, no. 6278, pp. 1176-1180, 2016.

[43] M. Kues, et al., "On-chip generation of high-dimensional entangled quantum states and their coherent control", Nature, vol. 546, no. 7660, pp. 622-626, 2017.

[44] P. Roztocki et al., "Practical system for the generation of pulsed quantum frequency combs," Optics Express, vol. 25, no. 16, pp. 18940-18949, 2017.

[45] Y. Zhang, et al., "Induced photon correlations through superposition of two four-wave mixing processes in integrated cavities", Laser and Photonics Reviews, vol. 14, no. 7, pp. 2000128, 2020. DOI: 10.1002/lpor.202000128

[46] C. Reimer, et al.,"High-dimensional one-way quantum processing implemented on d-level cluster states", Nature Physics, vol. 15, no.2, pp. 148-153, 2019.

[47] Stern, B., Ji, X., Okawachi, Y., Gaeta, A. L. \& Lipson, M. Battery-operated integrated frequency comb generator. Nature 562, 401 (2018).

[48] H. Bao, et al., Laser cavity-soliton microcombs, Nature Photonics, vol. 13, no. 6, pp. 384-389, Jun. 2019. 
[49]Lugiato, L. A., Prati, F. \& Brambilla, M. Nonlinear Optical Systems, (Cambridge University Press, 2015).

[50] Cole, D. C., Lamb, E. S., Del'Haye, P., Diddams, S. A. \& Papp, S. B. Soliton crystals in Kerr resonators. Nature Photonics 11, 671 (2017).

[51] Wang, W., et al.., Robust soliton crystals in a thermally controlled microresonator, Opt. Lett., 43, 2002 (2018).

[52] Bao, C., et al., Direct soliton generation in microresonators, Opt. Lett, 42, 2519 (2017).

[53] M.Ferrera et al., "CMOS compatible integrated all-optical RF spectrum analyzer", Optics Express, vol. 22, no. 18,21488 - 21498 (2014).

[54] A. Pasquazi, et al., "Sub-picosecond phase-sensitive optical pulse characterization on a chip", Nature Photonics, vol. 5, no. 10, pp. 618-623 (2011).

[55] M. Kues, et al., "Passively modelocked laser with an ultra-narrow spectral width", Nature Photonics, vol. 11, no. 3, pp. 159, 2017.

[56] L. Razzari, et al., "CMOS-compatible integrated optical hyper-parametric oscillator,” Nature Photonics, vol. 4, no. 1 , pp. 41-45, 2010.

[57] M. Ferrera, et al., "Low-power continuous-wave nonlinear optics in doped silica glass integrated waveguide structures," Nature Photonics, vol. 2, no. 12, pp. 737-740, 2008.

[58] M.Ferrera et al.“On-Chip ultra-fast $1^{\text {st }}$ and $2^{\text {nd }}$ order CMOS compatible all-optical integration”, Opt. Express, vol. 19, (23)pp. 23153-23161 (2011).

[59]D. Duchesne, M. Peccianti, M. R. E. Lamont, et al., "Supercontinuum generation in a high index doped silica glass spiral waveguide,” Optics Express, vol. 18, no, 2, pp. 923-930, 2010.

[60] H Bao, L Olivieri, M Rowley, ST Chu, BE Little, R Morandotti, DJ Moss, ... "Turing patterns in a fiber laser with a nested microresonator: Robust and controllable microcomb generation”, Physical Review Research 2 (2), 023395 (2020).

[61] M. Ferrera, et al., “On-chip CMOS-compatible all-optical integrator”, Nature Communications, vol. 1, Article 29, 2010.

[62] A. Pasquazi, et al., "All-optical wavelength conversion in an integrated ring resonator," Optics Express, vol. 18, no. 4, pp. 3858-3863, 2010.

[63] A. Pasquazi, Y. Park, J. Azana, et al., "Efficient wavelength conversion and net parametric gain via Four Wave Mixing in a high index doped silica waveguide," Optics Express, vol. 18, no. 8, pp. 7634-7641, 2010.

[64] M. Peccianti, M. Ferrera, L. Razzari, et al., "Subpicosecond optical pulse compression via an integrated nonlinear chirper," Optics Express, vol. 18, no. 8, pp. 7625-7633, 2010.

[65] Little, B. E. et al., "Very high-order microring resonator filters for WDM applications", IEEE Photonics Technol. Lett. 16, 2263-2265 (2004).

[66] M. Ferrera et al., "Low Power CW Parametric Mixing in a Low Dispersion High Index Doped Silica Glass Micro-Ring Resonator with Q-factor > 1 Million”, Optics Express, vol.17, no. 16, pp. 14098-14103 (2009).

[67] M. Peccianti, et al., "Demonstration of an ultrafast nonlinear microcavity modelocked laser", Nature Communications, vol. 3, pp. 765, 2012.

[68] A. Pasquazi, et al., "Self-locked optical parametric oscillation in a CMOS compatible microring resonator: a route to robust optical frequency comb generation on a chip," Optics Express, vol. 21, no. 11, pp. 13333-13341, 2013.

[69] A. Pasquazi, et al., "Stable, dual mode, high repetition rate mode-locked laser based on a microring resonator," Optics Express, vol. 20, no. 24, pp. 27355-27362, 2012.

[70] Wu, J. et al. RF Photonics: An Optical Microcombs' Perspective. IEEE Journal of Selected Topics in Quantum Electronics Vol. 24, 6101020, 1-20 (2018).

[71] Xu, X., et al., Photonic microwave true time delays for phased array antennas using a $49 \mathrm{GHz}$ FSR integrated micro-comb source, Photonics Research, 6, B30-B36 (2018).

[72] T. G. Nguyen et al., "Integrated frequency comb source-based Hilbert transformer for wideband microwave photonic phase analysis," Opt. Express, vol. 23, no. 17, pp. 22087-22097, Aug. 2015.

[73] X. Xu, J. Wu, M. Shoeiby, T. G. Nguyen, S. T. Chu, B. E. Little, R. Morandotti, A. Mitchell, and D. J. Moss, "Reconfigurable broadband microwave photonic intensity differentiator based on an integrated optical frequency comb source," APL Photonics, vol. 2, no. 9, 096104, Sep. 2017.

[74] X. Xu, M. Tan, J. Wu, R. Morandotti, A. Mitchell, and D. J. Moss, "Microcomb-based photonic RF signal processing", IEEE Photonics Technology Letters, vol. 31 no. 23 1854-1857, 2019.

[75] X. Xu, et al., "Broadband RF channelizer based on an integrated optical frequency Kerr comb source," Journal of Lightwave Technology, vol. 36, no. 19, pp. 4519-4526, 2018. 
[76] X. Xu, et al., "Continuously tunable orthogonally polarized RF optical single sideband generator based on micro-ring resonators," Journal of Optics, vol. 20, no. 11, 115701. 2018.

[77] X. Xu, et al., "Orthogonally polarized RF optical single sideband generation and dual-channel equalization based on an integrated microring resonator," Journal of Lightwave Technology, vol. 36, no. 20, pp. 4808-4818. 2018.

[78] M.Tan, X. Xu, J. Wu, B. Corcoran, A. Boes, T. G. Nguyen, Sai T. Chu, B. E. Little, R.Morandotti, A. Mitchell, and D. J. Moss, "Integral order photonic RF signal processors based on a soliton crystal micro-comb source", IOP Journal of Optics vol. 23 (11) 125701 (2021).

[79] X. Xu, et al., "Advanced adaptive photonic RF filters with 80 taps based on an integrated optical micro-comb source," Journal of Lightwave Technology, vol. 37, no. 4, pp. 1288-1295, 2019.

[80] X. Xu, et al., Broadband microwave frequency conversion based on an integrated optical micro-comb source", Journal of Lightwave Technology, vol. 38 no. 2, pp. 332-338, 2020.

[81] M. Tan, et al., "Photonic RF and microwave filters based on $49 \mathrm{GHz}$ and $200 \mathrm{GHz}$ Kerr microcombs", Optics Comm. vol. 465,125563, Feb. 22. 2020.

[82] X. Xu, et al., "Broadband photonic RF channelizer with 90 channels based on a soliton crystal microcomb", Journal of Lightwave Technology, Vol. 38, no. 18, pp. 5116 - 5121, 2020. doi: 10.1109/JLT.2020.2997699.

[83] X. Xu, et al., "Photonic RF and microwave integrator with soliton crystal microcombs", IEEE Transactions on Circuits and Systems II: Express Briefs, vol. 67, no. 12, pp. 3582-3586, 2020. DOI:10.1109/TCSII.2020.2995682.

[84] X. Xu, et al., "Photonic RF phase-encoded signal generation with a microcomb source", J. Lightwave Technology, vol. 38, no. 7, 1722-1727, 2020.

[85] X. Xu, et al., "High performance RF filters via bandwidth scaling with Kerr micro-combs," APL Photonics, vol. 4, no. 2, pp. 026102. 2019.

[86] M. Tan, et al., "Microwave and RF photonic fractional Hilbert transformer based on a 50 GHz Kerr microcomb", Journal of Lightwave Technology, vol. 37, no. 24, pp. 6097 - 6104, 2019.

[87] M. Tan, et al., "RF and microwave fractional differentiator based on photonics", IEEE Transactions on Circuits and Systems: Express Briefs, vol. 67, no.11, pp. 2767-2771, 2020. DOI:10.1109/TCSII.2020.2965158.

[88] M. Tan, et al., "Photonic RF arbitrary waveform generator based on a soliton crystal micro-comb source", Journal of Lightwave Technology, vol. 38, no. 22, pp. 6221-6226, Oct 22. 2020. DOI: 10.1109/JLT.2020.3009655.

[89] M. Tan, X. Xu, J. Wu, R. Morandotti, A. Mitchell, and D. J. Moss, "RF and microwave high bandwidth signal processing based on Kerr Micro-combs", Advances in Physics X, VOL. 6, NO. 1, 1838946 (2021). DOI:10.1080/23746149.2020.1838946.

[90] X. Xu, et al., "Advanced RF and microwave functions based on an integrated optical frequency comb source," Opt. Express, vol. 26 (3) 25692018.

[91] Kues, M. et al. Quantum optical microcombs. Nature Photonics 13, (3) 170-179 (2019). doi:10.1038/s41566019-0363-0

[92] P.Roztocki et al., "Complex quantum state generation and coherent control based on integrated frequency combs", Journal of Lightwave Technology 37 (2) 338-347 (2019).

[93] S. Sciara et al., "Generation and Processing of Complex Photon States with Quantum Frequency Combs", IEEE Photonics Technology Letters $\underline{31}$ (23) 1862-1865 (2019). DOI: 10.1109/LPT.2019.2944564.

[94] L. Caspani, C. Reimer, M. Kues, et al., "Multifrequency sources of quantum correlated photon pairs on-chip: a path toward integrated Quantum Frequency Combs," Nanophotonics, vol. 5, no. 2, pp. 351-362, 2016.

[95] C. Reimer et al., "Generation of multiphoton entangled quantum states by means of integrated frequency combs,” Science, vol. 351, no. 6278, pp. 1176-1180, 2016.

[96] M. Kues, et al., "On-chip generation of high-dimensional entangled quantum states and their coherent control", Nature, vol. 546, no. 7660, pp. 622-626, 2017.

[97] P. Roztocki et al., "Practical system for the generation of pulsed quantum frequency combs," Optics Express, vol. 25, no.16, 18940-18949, 2017.

[98] Y. Zhang, et al., "Induced photon correlations through superposition of two four-wave mixing processes in integrated cavities", Laser and Photonics Reviews, vol. 14, no. 7, pp. 2000128, 2020. DOI: 10.1002/lpor.202000128

[99] C. Reimer, et al., "High-dimensional one-way quantum processing implemented on d-level cluster states", Nature Physics, vol. 15 (2) 148 (2019). 
[100] H. Bao, et al., Laser cavity-soliton microcombs, Nature Photonics, vol. 13, no. 6, pp. 384-389, Jun. 2019.

[101] Bao, C., et al., Direct soliton generation in microresonators, Opt. Lett, 42, 2519 (2017).

[102] M. Ferrera et al., "CMOS compatible integrated all-optical RF spectrum analyzer", Optics Express, vol. 22, (18) 21488 (2014).

[103] A. Pasquazi, et al., "Sub-picosecond phase-sensitive optical pulse characterization on a chip", Nature Photonics, vol. 5, no. 10, pp. 618-623 (2011).

[104] M. Kues, et al., "Passively modelocked laser with an ultra-narrow spectral width", Nature Photonics, vol. 11, no. 3, pp. 159, 2017.

[105] L. Razzari, et al., "CMOS-compatible integrated optical hyper-parametric oscillator," Nature Photonics, vol. 4, no. 1, 41-45, 2010.

[106] M. Ferrera, et al., "Low-power continuous-wave nonlinear optics in doped silica glass integrated waveguide structures," Nature Photonics, vol. 2, no. 12, pp. 737-740, 2008.

[107] M.Ferrera et al.“On-Chip ultra-fast $1^{\text {st }}$ and $2^{\text {nd }}$ order CMOS compatible all-optical integration”, Opt. Express, vol. 19, (23)pp. 23153-23161 (2011).

[108] D. Duchesne, M. Peccianti, M. R. E. Lamont, et al., "Supercontinuum generation in a high index doped silica glass spiral waveguide," Optics Express, vol. 18, no, 2, pp. 923-930, 2010.

[109] H Bao, L Olivieri, M Rowley, ST Chu, BE Little, R Morandotti, DJ Moss, ... "Turing patterns in a fiber laser with a nested microresonator: Robust and controllable microcomb generation", Physical Review Research 2 (2), 023395 (2020).

[110] M. Ferrera, et al., "On-chip CMOS-compatible all-optical integrator”, Nature Communications, vol. 1, Article 29, 2010.

[111] A. Pasquazi, et al., "All-optical wavelength conversion in an integrated ring resonator," Optics Express, vol. 18 (4) 3858 (2010).

[112] A. Pasquazi, Y. Park, J. Azana, et al., "Efficient wavelength conversion and net parametric gain via Four Wave Mixing in a high index doped silica waveguide," Optics Express, vol. 18, no. 8, pp. 7634-7641, 2010.

[113] M. Peccianti, M. Ferrera, L. Razzari, et al., "Subpicosecond optical pulse compression via an integrated nonlinear chirper," Optics Express, vol. 18, no. 8, pp. 7625-7633, 2010.

[114] Little, B. E. et al., "Very high-order microring resonator filters for WDM applications", IEEE Phot. Technol. Lett. 16, 2263(2004).

[115] M. Ferrera et al., "Low Power CW Parametric Mixing in a Low Dispersion High Index Doped Silica Glass Micro-Ring Resonator with Q-factor > 1 Million", Optics Express, vol.17, no. 16, pp. 14098-14103 (2009).

[116] M. Peccianti, et al., "Demonstration of an ultrafast nonlinear microcavity modelocked laser", Nature Comm., vol. 3, 765, 2012.

[117] A. Pasquazi, et al., "Self-locked optical parametric oscillation in a CMOS compatible microring resonator: a route to robust optical frequency comb generation on a chip," Optics Express, vol. 21, no. 11, pp. 1333313341, 2013.

[118] A. Pasquazi, et al., "Stable, dual mode, high repetition rate mode-locked laser based on a microring resonator," Optics Express, vol. 20, no. 24, pp. 27355-27362, 2012.

[119] Xu, X., et al., Photonic microwave true time delays for phased array antennas using a 49 GHz FSR integrated micro-comb source, Photonics Research, 6, B30-B36 (2018).

[120] X. Xu, M. Tan, J. Wu, R. Morandotti, A. Mitchell, and D. J. Moss, "Microcomb-based photonic RF signal processing", IEEE Photonics Technology Letters, vol. 31 no. 23 1854-1857, 2019.

[121] M. Tan et al, "Orthogonally polarized Photonic Radio Frequency single sideband generation with integrated micro-ring resonators", IOP Journal of Semiconductors, Vol. 42 (4), 041305 (2021). DOI: 10.1088/16744926/42/4/041305.

[122] Xu, et al., "Advanced adaptive photonic RF filters with 80 taps based on an integrated optical micro-comb source," Journal of Lightwave Technology, vol. 37, no. 4, pp. 1288-1295 (2019).

[123] X. Xu, et al., Broadband microwave frequency conversion based on an integrated optical micro-comb source", Journal of Lightwave Technology, vol. 38 no. 2, pp. 332-338, 2020.

[124] M. Tan, et al., "Photonic RF and microwave filters based on 49GHz and 200GHz Kerr microcombs", Optics Comm. vol. 465,125563, Feb. 22. 2020.

[125] X. Xu, et al., "Broadband photonic RF channelizer with 90 channels based on a soliton crystal microcomb", Journal of Lightwave Technology, Vol. 38, no. 18, pp. 5116 - 5121, 2020. doi: 10.1109/JLT.2020.2997699. 
[126] X. Xu, et al., "Photonic RF and microwave integrator with soliton crystal microcombs", IEEE Transactions on Circuits and Systems II: Express Briefs, vol. 67, no. 12, pp. 3582-3586, 2020. DOI:10.1109/TCSII.2020.2995682.

[127] X. Xu, et al., "High performance RF filters via bandwidth scaling with Kerr micro-combs," APL Photonics, vol. 4 (2) 026102. 2019.

[128] M. Tan, et al., "Microwave and RF photonic fractional Hilbert transformer based on a $50 \mathrm{GHz}$ Kerr microcomb", Journal of Lightwave Technology, vol. 37, no. 24, pp. 6097 - 6104, 2019.

[129] M. Tan, et al., "RF and microwave fractional differentiator based on photonics", IEEE Transactions on Circuits and Systems: Express Briefs, vol. 67, no.11, pp. 2767-2771, 2020. DOI:10.1109/TCSII.2020.2965158.

[130] M. Tan, et al., "Photonic RF arbitrary waveform generator based on a soliton crystal micro-comb source", Journal of Lightwave Technology, vol. 38, no. 22, pp. 6221-6226 (2020). DOI: 10.1109/JLT.2020.3009655.

[131] M. Tan, X. Xu, J. Wu, R. Morandotti, A. Mitchell, and D. J. Moss, "RF and microwave high bandwidth signal processing based on Kerr Micro-combs”, Advances in Physics X, VOL. 6, NO. 1, 1838946 (2021). DOI:10.1080/23746149.2020.1838946.

[132] X. Xu, et al., "Advanced RF and microwave functions based on an integrated optical frequency comb source," Opt. Express, vol. 26 (3) 2569 (2018).

[133] M. Tan, X. Xu, J. Wu, B. Corcoran, A. Boes, T. G. Nguyen, S. T. Chu, B. E. Little, R.Morandotti, A. Lowery, A. Mitchell, and D. J. Moss, ""Highly Versatile Broadband RF Photonic Fractional Hilbert Transformer Based on a Kerr Soliton Crystal Microcomb", Journal of Lightwave Technology vol. 39 (24) 7581-7587 (2021).

[134] Bao, C., et al., Direct soliton generation in microresonators, Opt. Lett, 42, 2519 (2017).

[135] Yuning Zhang, Yang Qu, Jiayang Wu, Linnan Jia, Yunyi Yang, Xingyuan Xu, Baohua Jia, and David J. Moss, "Enhanced Kerr nonlinearity and nonlinear figure of merit in silicon nanowires integrated with 2D graphene oxide films", ACS Applied Materials and Interfaces, Vol. 12 (29) 33094-33103 (2020). DOI:10.1021/acsami.0c07852

[136] D.Moss, "11 Tera-FLOP/s photonic convolutional accelerator and deep learning optical neural networks", Research Square, (2021). DOI: https://doi.org/10.21203/rs.3.rs-493347/v1.

[137] Moss, David (2020): “11.0 Tera-FLOP/second photonic convolutional accelerator for deep learning optical neural networks", TechRxiv. Preprint. (2020). https://doi.org/10.36227/techrxiv.13238423.v1

[138] Xu, X.; Tan, M.; Corcoran, B.; Wu, J.; Boes, A.; Nguyen, T.; Chu, S.; Little, B.; Hicks, D.; Morandotti, R.; Mitchell, A.; Moss, D. “11 Tera-FLOP per Second Photonic Convolutional Accelerator for Deep Learning Optical Neural Networks", Preprints 2020, 2020110420.

[139] Moss, David (2020): "RF and microwave photonic high bandwidth signal processing based on Kerr microcomb sources", TechRxiv. (2020). Preprint. DOI:10.36227/techrxiv.12665609.v3

[140] Yuning Zhang, Jiayang Wu, Yunyi Yang, Yang Qu, Linnan Jia, Tania Moein, Baohua Jia, David J. Moss, "Enhanced nonlinear optical figure-of-merit at $1550 \mathrm{~nm}$ for silicon nanowires integrated with graphene oxide layered films", Arxiv (2020). arXiv:2004.08043 [physics.optics]

[141] Moss, David; Jia, Baohua; Wu, Jiayang; Zhang, Yuning; Yang, Yunyi; Jia, Linnan, Yang Qu, Tania Moein (2020): “Transforming silicon into a high performing integrated nonlinear photonics platform by integration with 2D graphene oxide films", TechRxiv. (2020). Preprint. DOI:10.36227/techrxiv.12061809.v1.

[142] A. Frigg, A. Boes, G. Ren, T.G. Nguyen, D.Y. Choi, S. Gees, D. Moss, A Mitchell, “Optical frequency comb generation with low temperature reactive sputtered silicon nitride waveguides", APL Photonics, Vol. 5 (1), 011302 (2020).

[143] T. Moein, D. Gailevičius, T. Katkus, S.H. Ng, S. Lundgaard, D.J. Moss, H. Kurt, Vygantas Mizeikis, Kȩstutis Staliūnas, Mangirdas Malinauskas, Saulius Juodkazis, "Optically-thin broadband graphene-membrane photodetector”, Nanomaterials, Vol. 10 (3), 407 (2020).

[144] M. Tan, X. Xu, J. Wu, A. Boes, B. Corcoran, T. G. Nguyen, S. T. Chu, B. E. Little, R. Morandotti, A. Mitchell, and D. J. Moss, “Advanced applications of Kerr mircocombs”, Paper 11775-1. SPIE 11775, Integrated Optics: Design, Devices, Systems and Applications VI, (EOO21) OO107-8, Proc 1177504 (18 April 2021); Integrated Optics Conference, SPIE Optics and Optoelectronics Symposium, Prague, Czech Republic. April 19 - 22 (2021), doi.org/10.1117/12.2588733.

[145] Moss, David, "Microcombs for Ultrahigh Bandwidth Optical Data Transmission and Neural Networks." OSF Preprints. March 8. (2021). DOI:10.31219/osf.io/ne9wx. 